Article

\title{
Optical Sideband Injection Locking Using Waveguide Based External Cavity Semiconductor Lasers for Narrow-Line, Tunable Microwave Generation
}

\author{
Md. Rezaul Hoque Khan 1,2,* (D) and Md. Ashraful Hoque ${ }^{1}$ \\ 1 Islamic University of Technology, Dhaka, Gazipur 1704, Bangladesh \\ 2 Telecommunication Engineering Group, Faculty of EEMCS, University of Twente, \\ 7500 AE Enschede, The Netherlands \\ * Correspondence: rhkhan@iut-dhaka.edu
}

Received: 18 June 2019; Accepted: 12 July 2019; Published: 20 July 2019

\begin{abstract}
The generation by optical injection locking of spectrally unadulterated microwave signals using waveguide based external cavity semiconductor lasers (WECSL) is demonstrated. A tunable frequency of $2-11 \mathrm{GHz}$, limited by the modulator's bandwidth and the photodetector (PD), was created as proof-of-experiment by the injection locking of the two WESCLs. A single sideband (SSB) phase noise of $-75 \mathrm{dBc} / \mathrm{Hz}$ from the generated carrier at $10 \mathrm{kHz}$ offset and a phase noise variance at an optimum injection ratio region was $0.03 \mathrm{rad}^{2}$, corresponding to $1.7^{\circ}$, were observed. The main feature of this approach is the consolidation of the upsides of microwave generation at low phase noise with a broad tuning range and the capacity of hybrid photonic integration. In addition, the injection locking characteristics were used to determine the $Q$ factor of the complicated optical cavities with unknown inner losses.
\end{abstract}

Keywords: optical injection locking; microwave carrier generation; hybrid photonic integration; locking range

\section{Introduction}

Recently, photonic production and distribution of microwave carriers attract a wide interest due to its massive ability for distribution and noticeably very high-frequency operation [1-3], utilizing methods based on optical frequency combs, mode-locked lasers, heterodyne optical phase-locked loops and sideband-injection locking. Frequency-comb generators typically require a large, stabilized cavity, yielding systems that are comparatively bulky and complex [4]. The large number of modes and the small mode-spacing emitted by the mode-locked laser needs to be filtered before injecting the slave laser, otherwise it may prevent the single-mode stability of injection lock [5]. In heterodyning between two separate single frequency lasers, all the phase noise from each laser is directly transferred into the microwave carrier. Moreover, such scheme is rather bulky and suffers from large frequency drift of the microwave carrier [2]. Optical side frequency injection locking offers such a capability; the optical phase noise generated by spontaneous emission cancels when a slave laser is injected with a side frequency derived from the master laser.

Optical injection locking technique has generally been utilized to create narrow linewidth microwave carriers employing distributed feedback (DFB) semiconductor lasers [6,7], fiber lasers [8] or external cavity diode lasers (ECDL) [9]. Customarily, two lasers are utilized in an optical injection locking procedure. Light is injected from one laser, termed as the master laser, into the other laser, termed as the slave laser. At the point when the frequency of the slave laser is guided adjoining the frequency of the master laser, the slave laser begins lasing on that of the master laser. This occasion is called optical injection locking. 
Most of the optical methods presented in carrier generation in the previous works were centered around extensive tunability and low phase noise of the generated carriers still require minimization. The likelihood to consolidate different optical parts by means of photonic integration is generally considered as one of the key empowering advancements for what is to come [10]. Photonic integration technology attracts a great deal of attention due to its potential benefits concerning its cost-effective volume production and small footprint [11].

A diode laser has recently been reported where an optical gain chip has been coupled to an external cavity that has been integrated into a waveguide chip. This chip was fabricated using $\mathrm{Si}_{3} \mathrm{~N}_{4} / \mathrm{SiO}_{2}$ waveguide technology (TriPleX ${ }^{T M}$ ) with a box shaped cross section [12]. The optical gain chip joined to the waveguide chip was alluded as waveguide based external cavity semiconductor laser (WECSL) [13]. With TriPleX ${ }^{T M}$, higher integration levels and hybrid combinations with other commercially available platforms (for instance InP and SOI) are also possible through on-chip, low loss spot-size convertors. As the WECSL is essentially a filter cavity with a gain medium, it can make use of the great potential of hybrid photonic integration.

External cavity lasers are known to offer an astounding overall performance regarding its optical power $(\mathrm{mW})[14,15]$ linewidth $(\mathrm{kHz})$ [13] and tuning range $(\mathrm{THz})$ [14] rather than different sorts of of lasers like the DFB lasers [16] or external cavity diode lasers (ECDL) [9]. It is recommended that lasers possess large frequency tunability to have great potential in optical injection locking scheme [8]. Moreover, an integrated laser module would be suitable for most well known optical injection locking scheme, namely the side frequency injection locking scheme, explained in detail in [7,11]. In this paper, a glass-based waveguide circuit to create a hybrid semiconductor-glass laser has a great perspective to be used in a side frequency injection locking scheme for the first time.

The organization of this paper is as follows. Section 2 presents the characteristic of the WECSL used for the injection-locked loop. Section 3 gives the optical injection locking principle. Sections 4 and 5 provide the experimental demonstration and experimental results, respectively. A summary is presented at the end of this paper.

\section{Characteristic of The Wecsl}

The detailed characterization of the WECSL used in our scheme was presented by $R$. M. Oldenbeuving et al. [13]. In a WECSL, an optical "gain chip", developed in Fraunhofer Heinrich-Hertz-Institut [13], is integrated to an outer mirror coordinated into a waveguide chip, as shown in Figure 1.

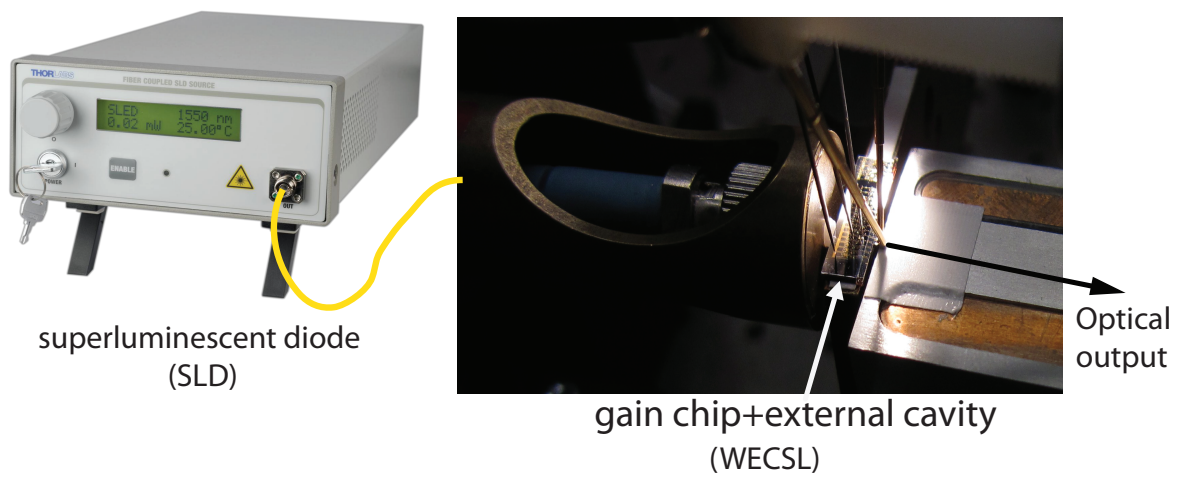

Figure 1. Photograph of the WECSL setup.

The schematic outline of the entire waveguide chip is shown in Figure 2. The waveguide chip accommodates a dual micro-ring resonator (MRR) structure [17], termed as an MRR mirror, which can be combined with a directional couplers. This magnificent combination acts as a frequency 
selective mirror. Moreover, a bi-directional coupler launches light from a straight waveguide into a "measurement channel" in order to monitor the performance of the laser and mirror.

(a)

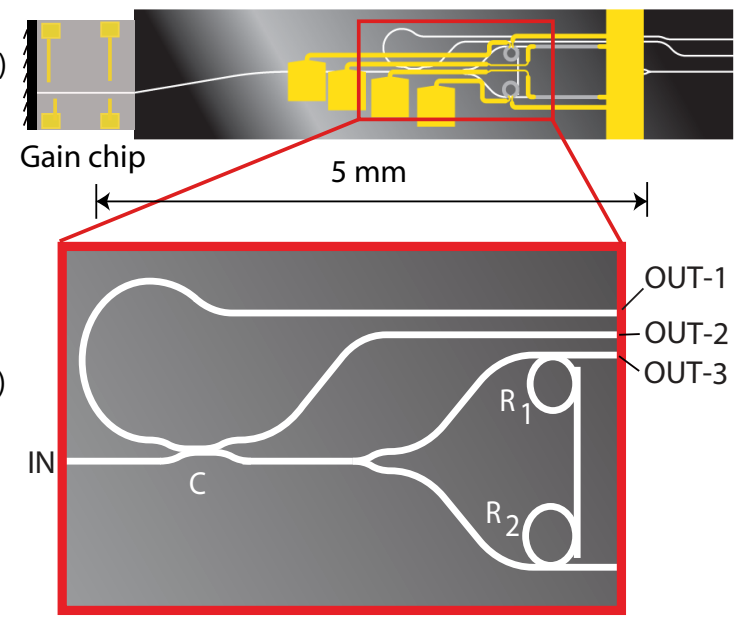

Figure 2. Schematic of the tunable reflector waveguide chip. (a) Complete chip of the waveguide The waveguides are depicted in white, electrical contacts are yellow, and the heaters are grey. (b) Dual micro-ring resonators, $R_{1}$ and $R_{2}$, where " $C$ " is marked as coupler, "IN" is marked as the input port of the waveguide chip, "OUT -1 " and "OUT -2 " are marked as output ports of the computation channel, and "OUT - 3" marks the output port of the WECSL. (Reproduced with modification by permission from Laser Physics Letters [13]).

To characterize the mirror, the spectrum from 1500 to $1600 \mathrm{~nm}$ from a superluminescent diode (SLD) (Thorlabs S5FC1005S) was inserted to the input port of the waveguide chip ("IN" port in Figure 2). The MRR-mirror's response was measured using a fiber coupled SLD and butt-coupled via a PM-fiber to the input port on the waveguide chip. The reflected spectrum of the MRR mirror is measured at the output port of the measurement channel ("OUT-1" port of Figure 2) using an optical spectrum analyzer (OSA). The resonant frequency of the highest peak of the MRR corresponds to the lasing frequency, which is tuned by shifting the resonant frequency, i.e., via thermo optical effect by heating the MRRs. The WECSL can be tuned over the entire telecommunication C band region (1530-1565 nm) [13].

\section{Side Frequency Injection Locking}

Different strategies proposed to date depending on optical injection locking techniques and side frequency injection locking can produce unadulterated microwave carriers. Moreover, the framework dependent on it has shown high stability and tunability for the carriers [18]. The mechanisms of the side frequency injection locking technique are discussed in this section. The master laser light is divided into two parts. One part of the light is frequency modulated to form optical side frequencies and one of the side frequencies is chosen by a filter and infused into the slave laser. The slave laser is injection-locked onto the side frequency of the master laser. The other part of the light from the master laser is heterodyned with the light from the injection-locked slave laser to produce the microwave carrier. The schematic of this lockup mechanism is shown in Figure 3. 


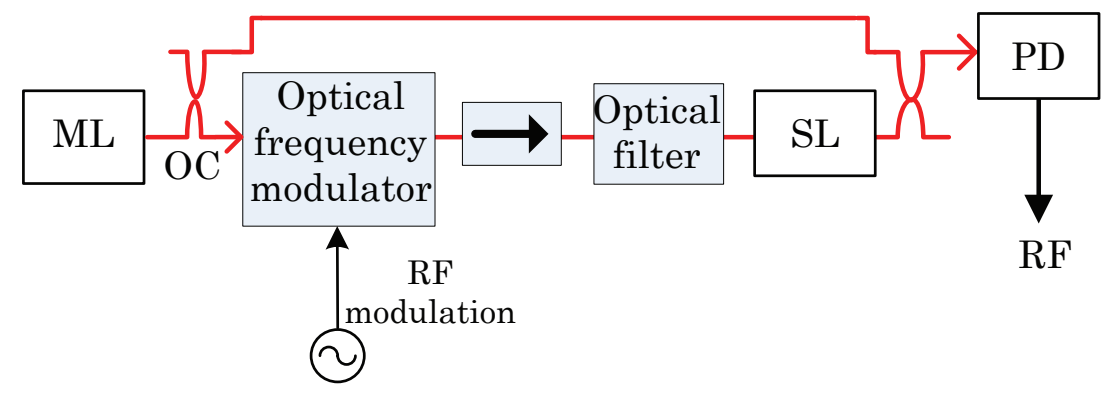

Figure 3. Schematic of side frequency injection locking. ML, master laser; OC, optical coupler; OFM, optical frequency modulator; SL, slave laser; PD, photodetector.

As described in [19], the frequency range in which the frequency of the slave laser turn out to be rigidly settled to the side frequency of the master laser is called the injection locking range or locking bandwidth. The locking extent can be dictated by concurrent tuning the side frequency of the master laser and checking the RF beat spectrum. At the point when the side frequency of the master laser is tuned towards the frequency of the free-running slave laser, the slave laser suddenly begins to sway along the side frequency of the master laser and injection-locked beat spectrum appears. Tuning the side frequency away from the slave laser breaks the locking. While in locking condition, the laser dynamics of the slave laser are governed by the infused light. However, the locking range is administered by the infusion proportion and the quality of the laser cavity [20]. The infusion proportion is characterized as the proportion between the infused optical power from the side frequency of the master laser and the optical yield of the free-running slave laser. The injection locking bandwidth, $\Delta v_{\text {lock}}$, is expressed as [19]

$$
\Delta v_{\text {lock }}=\frac{v_{0}}{Q} \sqrt{\frac{P_{\text {inj }}}{P_{\mathrm{s}}}}
$$

where $v_{0}$ is the frequency of the light output of the slave laser, $Q$ is the quality factor of the slave lasers cavity, $P_{\text {inj }}$ is the injection power of the side frequency of the master laser and $P_{\mathrm{s}}$ is the output power of the slave laser. The infusion proportion $R$ is characterized as the proportion between the externally infused optical power, $P_{\text {inj, }}$ and that discharged from the slave laser, $P_{\mathrm{s}}$. In [19], an articulation for half of the locking bandwidth was introduced. The articulation in Equation (1) is modified to represent the full locking bandwidth.

\section{Experimental Demonstration}

An experiment was carried out to verify the proposed approach. The schematic of the employed experimental setup is shown in Figure 4. 


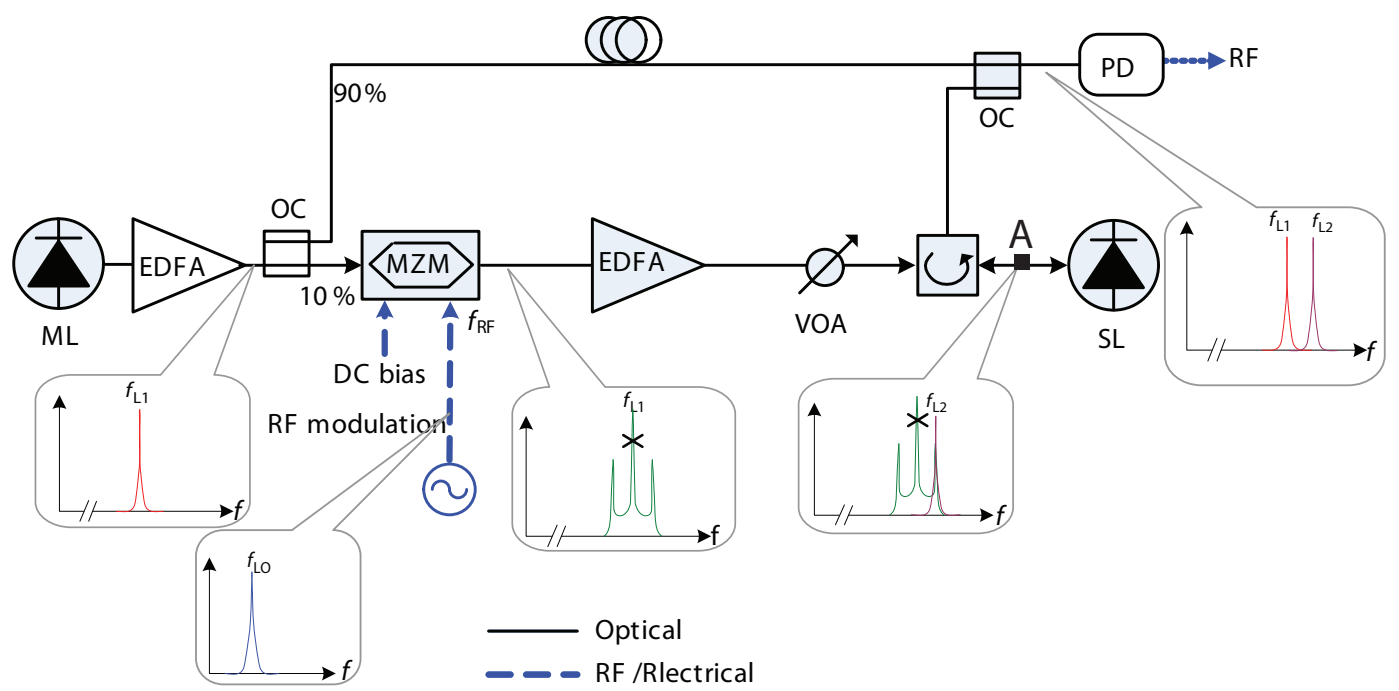

Figure 4. Schematic of the experimental setup for injection locking of WECSLs. Highlights point out the spectra at various points. ML, master laser; OC, optical coupler; MZM, Mach-Zehnder modulator; EDFA, erbium doper fiber amplifier; VOA, variable optical attenuator; SL, slave laser; PD, photo-detector. The optical power from the master laser is injected into the slave laser at Point A.

The waveguide chips of the master and slave lasers were butt-coupled to single mode fibers (SMFs). Two identical but separate WECSL lasers were used as a master laser and slave laser. The center frequencies of both lasers were around 194.05 THz. Four Erbium doped fiber amplifiers (EDFAs) were used from two different manufacturers, namely Alcatel (type 1686WM) and Firmstein Technologies Inc. (type PR25R). The fiber-coupled optical power of the master laser was measured to be $18.8 \mathrm{dBm}$. The amplified output power of the master laser was coupled out via 10\% port of the 10:90 optical coupler (OC). Its output was modulated by a Mach-Zehnder modulator (MZM) (Avanex powerLog FA 20) biased at its half wave voltage $V_{\pi}=+5 \mathrm{~V}$, and modulated by a $20 \mathrm{dBm}$ RF reference signal . The values of the bias voltage and the RF reference signal were chosen to suppress the optical carrier and to maximize the modulation side frequencies with first-order harmonic suppression of $8.2 \mathrm{~dB}$. The output light from the modulator was passed through an isolator and again amplified using two EDFAs. Afterwards, the light passed through a variable optical attenuator (VOA) before it was split in a $3 \mathrm{~dB}$ coupler. The optical output from the $3 \mathrm{~dB}$ coupler was injected into the slave laser through an SMF. The fiber-coupled optical power of the free-running slave laser and the master laser before inserting to the slave laser (at Point A in Figure 4) were measured to be $-16.8 \mathrm{dBm}$ and $4.3 \mathrm{dBm}$, respectively. The total optical power at the positive first-order side frequency was $3.9 \mathrm{dBm}$. The remaining $90 \%$ optical power from the 10:90 coupler and the amplified output of the slave laser from the $3 \mathrm{~dB}$ coupler was combined in another $3 \mathrm{~dB}$ coupler, which finally went to a PD (Discovery Semiconductor DSC20S) and a microwave beat signal was observed using an RF spectrum analyzer (Agilent MXA N9020A). The optical signals were detected by an optical spectrum analyzer (Ando AQ6317) with a resolution of $0.01 \mathrm{~nm}$. In this experiment, the wavelength and injection current of the optical carrier from the master laser were set to $1532.43 \mathrm{~nm}$ and $56 \mathrm{~mA}$, respectively. The first-order modulation side frequency at the output of the MZM was tuned from 2 to $12 \mathrm{GHz}$ using an RF reference. The natural wavelength of the slave laser was fine tuned so that it locked to the first-order side frequency. The slave laser can be tuned by either heating the MRR or injection current of the WECSL.

\section{Experimental Results}

In this section, we present the experimental investigation of the side frequency injection locking technique. We also investigated the performance of the injection-locked technique by measuring various parameters such as the locking range and phase noise of the injection-locked WECSL. 


\subsection{Locking Range}

In the experiment, the injected power was attenuated with steps of $0.5 \mathrm{~dB}$ using a VOA. For each injected power, the locking range was measured by sweeping the side frequency of the master laser such that the sweeping range was higher than the full locking bandwidth, as shown in Figure 5. When the side frequency of the master laser is outside of the locking bandwidth, multiple beat signals would appear at the output of the PD, as shown in Figure 5a,d. As soon as the side frequency of the master laser comes within the locking bandwidth, injection lock occurs and unadulterated beat signal is generated. Moving the side frequency of the master laser within this locking range would not break this lock, as shown in Figure 5b,c.

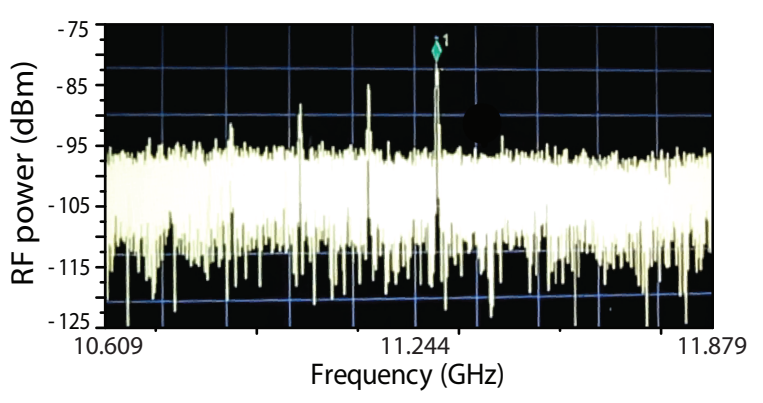

(a)

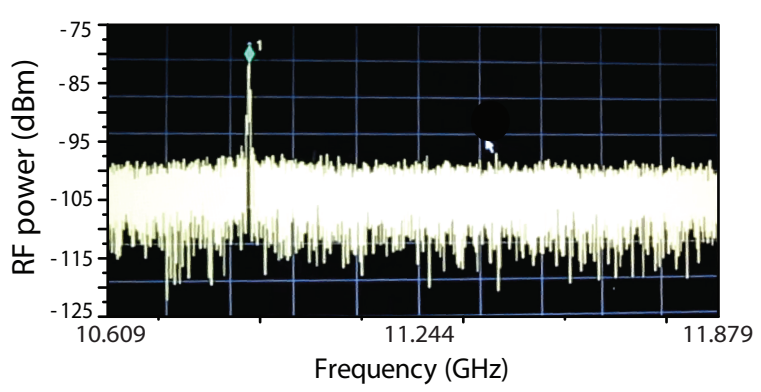

(c)

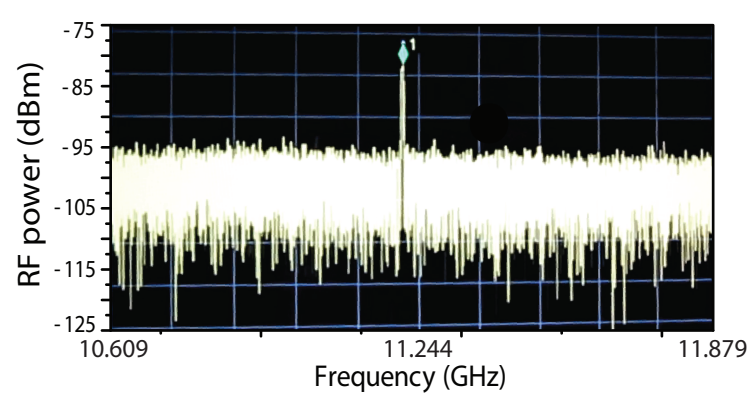

(b)

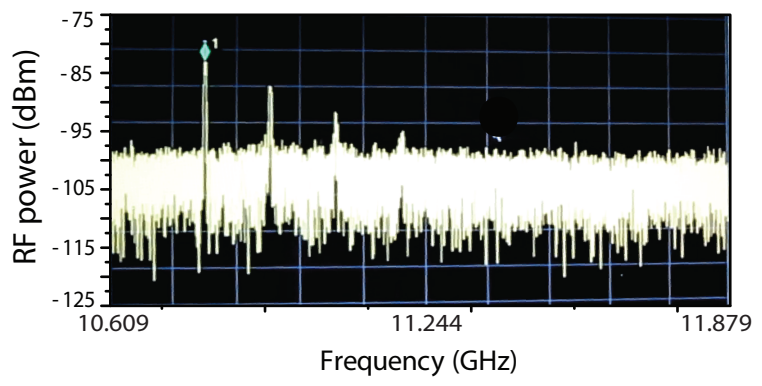

(d)

Figure 5. Injection locking range measurement for an injection power of $-18.7 \mathrm{dBm}$ : multiple beat signals indicate no locking $(\mathbf{a}, \mathbf{d})$; and single beat signal indicated locking occurs $(\mathbf{b}, \mathbf{c})$.

The optical power of the slave laser (without injection locking) before coupling with the circulator (at Point $\mathrm{A}$ in Figure 4 ) was measured by the OSA to be $P_{\mathrm{S}-\text { measured }}=-16.8 \mathrm{dBm}$. The amplified optical power of the master laser through the circulator was measured to be $P_{\text {inj-measured }}=4.3 \mathrm{dBm}$. The design of the waveguide cross section used in this experiment has a mode field diameter (MFD) of $0.9 \mu \mathrm{m} \times 1.3 \mu \mathrm{m}$, which is smaller than that of the MFD of the single mode fiber (SMF) of $10.5 \mu \mathrm{m}$ (www.thorlabs.com). Due to the modal mismatch between the MFD of the waveguide and the MFD of the SMF, the infusion power of the side frequency of the master laser, $P_{\text {inj }}$ and the output power of the slave laser, $P_{\mathrm{S}}$ must be calculated considering coupling efficiency between the SMF and the waveguide. Assuming no lateral and angular misalignments of the fiber axis relative to the waveguide axis and no space between the end-faces of the SMF and the waveguide, the coupling efficiency between the waveguide and the SMF is calculated as [21]

$$
\eta=\frac{4}{\left(\frac{\omega_{0 x}}{\omega_{1}}+\frac{\omega_{1}}{\omega_{0 x}}\right)\left(\frac{\omega_{0 y}}{\omega_{1}}+\frac{\omega_{1}}{\omega_{0 y}}\right)}
$$

where $\omega_{0 x}$ and $\omega_{0 y}$ are the MFD of the waveguide in the $\mathrm{x}$ and $\mathrm{y}$ axes, respectively, and $\omega_{1}$ is the MFD of the SMF. Using the values of MFD of the waveguide and the SMF in Equation (2), the coupling efficiency, $\eta$, was calculated as $4 \%(-14 \mathrm{~dB})$. Thus, the actual power injected from the side frequency 
(considering $15 \%$ or $-8.3 \mathrm{~dB}$ power fraction in the side frequency compared to the center frequency) of the master laser is $P_{\mathrm{inj}}=-18.6 \mathrm{dBm}$ and the actual output power of the slave laser is $P_{\mathrm{s}}=-2.4 \mathrm{dBm}$. Figure 6 shows the expected locking characteristics for the WECSL.

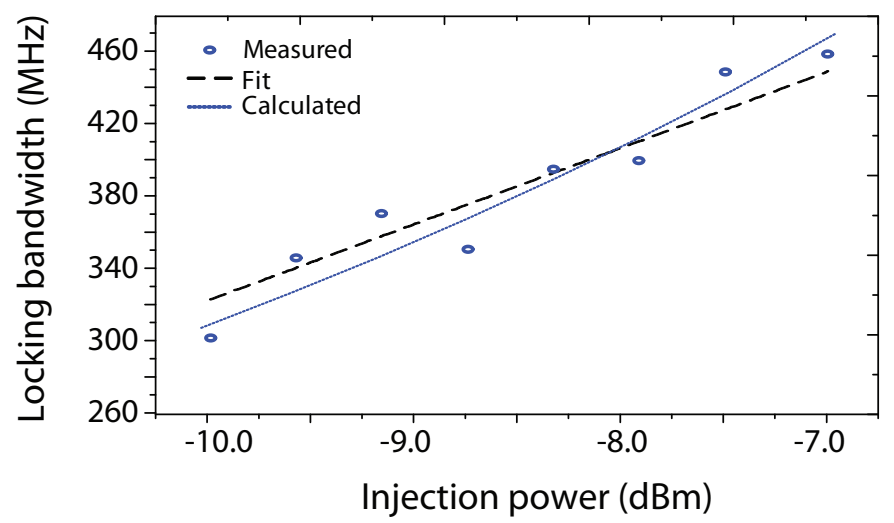

Figure 6. Experimental measurement and theoretical calculation of locking bandwidth versus injection power. The circle indicates the measured values. The dashed line indicates the theoretical fit for a $Q$ value of $6.2 \times 10^{4}$.

The locking bandwidth increases with an increase of injection power (or injection ratio, $R$ ), or decreasing the attenuation. No locking was observed below an injection ratio of $-21.2 \mathrm{~dB}$. The experimental measurements of locking bandwidths are compared with the theoretical calculation from Equation (1) and also plotted in Figure 6. The theoretical value of the $Q$ factor for the WECSL was calculated as $1.6 \times 10^{4}$ [13]. The fit curve in Figure 6 is plotted for a $Q$ value of $6.2 \times 10^{4}$. This leads to a calculated laser linewidth of $148 \mathrm{kHz}$, which is comparable to the measured laser linewidth of $25 \mathrm{kHz}$ [13] and shows a good agreement with the $Q$ factor determined by calculation.

\subsection{Phase Noise}

To investigate the improvement of the beat linewidth the phase noise measurements of the generated carriers were performed. The phase noise of the generated carrier was measured for both the free running (red line) and the injection-locked (black line) cases, and the results are shown in Figure 7.

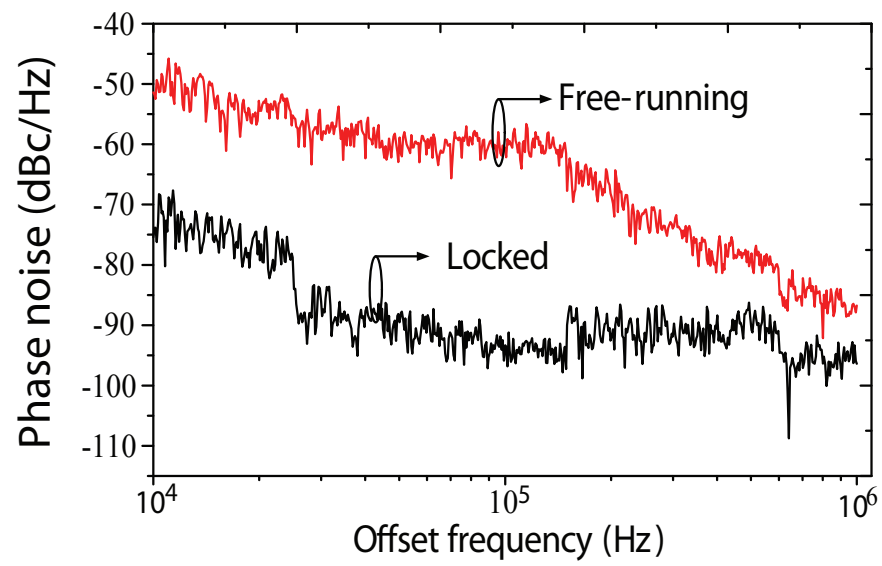

Figure 7. Phase noise of the beat signal in free-running and injection-locked measurement at the modulation frequency of $10 \mathrm{GHz}$.

The operating conditions (i.e., injection current and temperature) of the individual WECSL were kept unchanged during both the free-running and injection-locked measurements. When the slave laser was successfully injection-locked, a beat signal of $10 \mathrm{GHz}$ was observed with a single sideband 
(SSB) phase noise of $-75 \mathrm{dBc} / \mathrm{Hz}$ at $10 \mathrm{kHz}$ frequency offset from the carrier. This gives a $25 \mathrm{~dB}$ lower SSB phase noise compared to the free running measurement. The phase noise variance for the injection-locked carrier was calculated by following the procedure in [22] by integrating the spectral density of the phase noise from offset frequencies $10 \mathrm{kHz}$ to $1 \mathrm{MHz}$. With an optimum injection ratio, the phase noise variance is $0.03 \mathrm{rad}^{2}$, corresponding to $1.7^{\circ}$.

\subsection{Frequency Stability}

Injection locking of the slave WESCL with the sideband of the master WESCL significantly improves its frequency stability. An injection ration of $22 \mathrm{~dB}$ was used for frequency stability evaluation of the injection lock loop. The measured frequency stability during a period of one minute with the MaxHold function of RF-SA is shown in Figure 8.

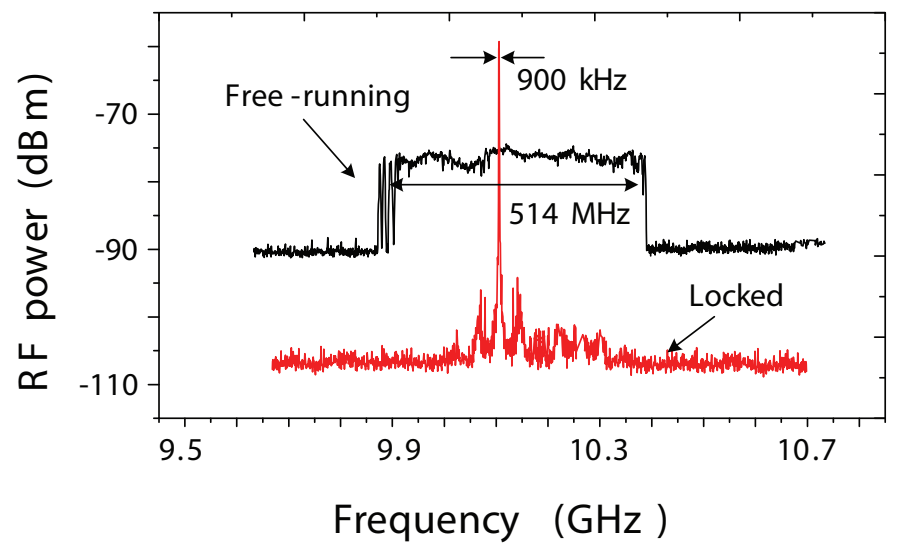

Figure 8. Measured frequency stability during a period of one minute with the MaxHold function of the RF-SA.

The standard deviation of the injection-locked microwave frequency during this period was found to be $900 \mathrm{kHz}$. In free running condition, this stability degraded to $514 \mathrm{MHz}$. The operating conditions (i.e., injection current, temperature) of each WESCL were exactly the same for the both free-running and injection-locked condition. By applying a voltage as it were to the ring heater with radius $R_{1}=5.0 \mathrm{~nm}$, the wavelength switches of the ring's FSR estimate with radius $R_{2}$ (i.e., $4.0 \mathrm{~nm}$ ) are anticipated to alter. For a voltage step from $0 \mathrm{~V}$ to $2.3 \mathrm{~V}$, a stable and reproducible wavelength switch was noted from $\lambda=1552.2 \mathrm{~nm}$ to $\lambda=1548.2 \mathrm{~nm}$ [13].

\subsection{Frequency Tunability}

As mentioned above, the external cavity of the WECSL used in the demonstration is a tunable micro-ring resonator (MRR) mirror. The MRR's resonance frequencies can be tuned by heating the MRR, resulting in faster tuning than tuning by injection current. The continuous frequency tuning of the injection-locked loop was investigated, while observing the beat signal of the two lasers. Wavelength tuning mechanism, heating of MRRs to modify their refractive index, is relatively coarse and it was implemented by injecting the generating optical side frequencies using a MZM. The RF reference signal for the MZM is provided by an RF oscillator (Avanex PowerLog FA 20), which can be tuned with a sub-kHz precision over a wide range (a few $\mathrm{kHz}$ to $20 \mathrm{GHz}$ ) of frequencies. This results in tuning the optical side frequencies, which ultimately tunes the microwave beat signal (as shown in Figure 9) from 2 to $11 \mathrm{GHz}$ with a sub-kHz precision. 


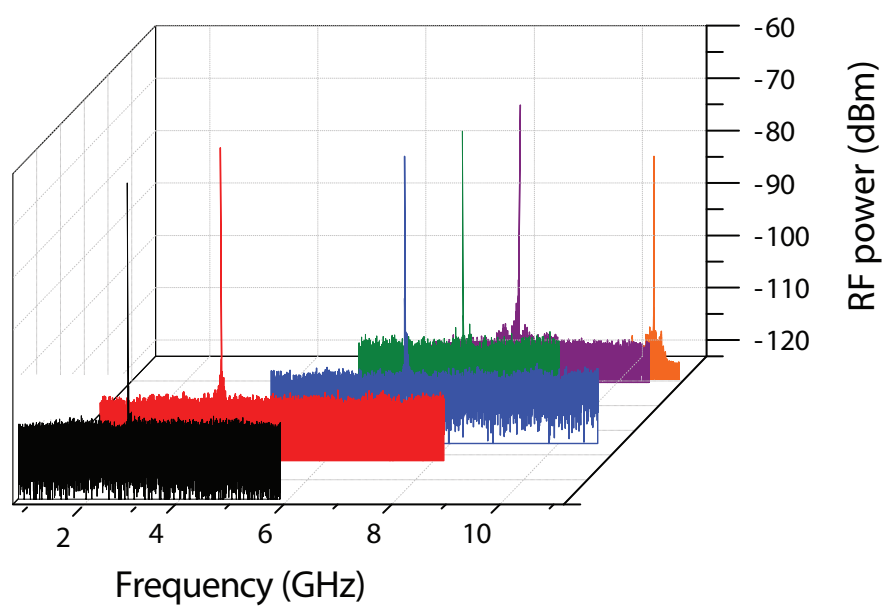

Figure 9. The spectra of the generated microwave carrier tuned from 2 to $11 \mathrm{GHz}$.

Because of the limited bandwidth of the PD, we could not observe frequencies higher than 11.2 GHz signal.

\section{Conclusions}

Generation of an unadulterated microwave signal using WECSL by means of optical injection locking is experimentally demonstrated. These measurements show that the injection locking behavior of the WECSL agrees with the existing theory on injection locking. The locking range of the injection-locked loop is also demonstrated. The phase noise performance of the generated microwave carrier was also observed. An SSB phase noise of $-75 \mathrm{dBc} / \mathrm{Hz}$ was observed at a $10 \mathrm{kHz}$ offset from the generated microwave carrier. It was found that the phase noise variance at an optimum injection ratio region was $0.03 \mathrm{rad}^{2}$, corresponding to $1.7^{\circ}$, which is suitable for many applications where a very low phase noise of the generated carriers are required such as in a satellite reception system. The experimental tuning range is obliged by the working scope of the modulator and the PD. The development of modulators capable of operating up to $300 \mathrm{GHz}$ [23] and PD facilitate in producing frequencies exceeding $500 \mathrm{GHz}$ [24] has already been reported. By properly choosing the higher-order side frequency of the master laser and optical injection locking the slave laser on that frequency, $\mathrm{PD}$ output as large as hundreds of $\mathrm{GHz}$ is obtainable, which may find application in radio over fiber system, medical imaging and spectroscopy in the pharmaceutical industry.

Author Contributions: Investigation, M.R.H.K.; and Supervision, M.A.H.

Funding: This research received no external funding.

Acknowledgments: The authors gratefully acknowledge the support of the Smart Mix Programme of the Netherlands Ministry of Economic Affairs and the Netherlands Ministry of Education, Culture and Science and Laser Physics and Nonlinear Optics (LPNO) group of University of Twente, The Netherlands for experimentation.

Conflicts of Interest: The authors declare no conflict of interest.

\section{References}

1. Ryu, H.Y.; Lee, S.H.; Suh, H.S. Widely Tunable External Cavity Laser Diode Injection Locked to an Optical Frequency Comb. IEEE Photon. Technol. Lett. 2010, 22, 1066-1068. [CrossRef]

2. Khan, M.; Islam, M.; Sarowar, G.; Reza, T.; Hoque, M. Carrier generation using a dual-frequency distributed feedback waveguide laser for phased array antenna (PAA). J. Eur. Opt. Soc.-Rapid Publ. 2017, 13, 30. [CrossRef]

3. Khan, M.R.; Bernhardi, E.H.; Marpaung, D.A.; Burla, M.; de Ridder, R.M.; Worhoff, K.; Pollnau, M.; Roeloffzen, C.G. Dual-frequency distributed feedback laser with optical frequency locked loop for stable microwave signal generation. IEEE Photonics Technol. Lett. 2012, 24, 1431-1433. [CrossRef] 
4. Hugi, A.; Villares, G.; Blaser, S.; Liu, H.; Faist, J. Mid-infrared frequency comb based on a quantum cascade laser. Nature 2012, 492, 229. [CrossRef]

5. Li, X.; Jeon, C.; Pan, S.; Kim, J. Low-Noise Repetition-Rate Multiplication by Injection Locking and GainSaturated Amplification. IEEE Photonics Technol. Lett. 2019, 31, 997-1000. [CrossRef]

6. Hui, R.; D'Ottavi, A.; Mecozzi, A.; Spano, P. Injection locking in distributed feedback semiconductor lasers. IEEE J. Quantum Electron. 1991, 27, 1688-1695. [CrossRef]

7. Schneider, G.J.; Murakowski, J.A.; Schuetz, C.A.; Shi, S.; Prather, D.W. Radiofrequency signal-generation system with over seven octaves of continuous tuning. Nat. Photonics 2013, 7, 118-122. [CrossRef]

8. Pan, S.; Tang, Z.; Zhu, D.; Ben, D.; Yao, J. Injection-locked fiber laser for tunable millimeter-wave generation. Opt. Lett. 2011, 36, 4722-4724. [CrossRef]

9. Saliba, S.D.; Scholten, R.E. Linewidths below $100 \mathrm{kHz}$ with external cavity diode lasers. Appl. Opt. 2009, 48, 6961-6966. [CrossRef]

10. Paniccia, M. Integrating silicon photonics. Nat. Photonics 2010, 4, 498-499.

11. Grund, D.W., Jr.; Schneider, G.J.; Ejzak, G.A.; Murakowski, J.; Shi, S.; Prather, D.W. Integrated silicon-photonic module for generating widely tunable, narrow-line RF using injection-locked lasers. In $R F$ and Millimeter-Wave Photonics II; International Society for Optics and Photonics: Bellingham, WA, USA , 2012; Volume 8259. [CrossRef]

12. Morichetti, F.; Melloni, A.; Martinelli, M.; Heideman, R.G.; Leinse, A.; Geuzebroek, D.H.; Borreman, A. Box-shaped dielectric waveguides: A new concept in integrated optics? J. Light. Technol. 2007, 25, 2579-2589. [CrossRef]

13. Oldenbeuving, R.M.; Klein, E.J.; Offerhaus, H.L.; Lee, C.J.; Song, H.; Boller, K.J. 25 kHz narrow linewidth of a wavelength tunable diode laser with a short waveguide-based external cavity. Laser Phy. Lett. 2013, 10, 1-8.

14. Fujioka, N.; Chu, T.; Ishizaka, M. Compact and Low Power Consumption Hybrid Integrated Wavelength Tunable Laser Module Using Silicon Waveguide Resonators. J. Lightw. Technol. 2010, 28, 3115-3120. [CrossRef]

15. Chu, T.; Fujioka, N.; Ishizaka, M. Compact, lower-power-consumption wavelength tunable laser fabricated with silicon photonic wire waveguide micro-ring resonators. Opt. Express 2009, 17, 14063-14068. [CrossRef]

16. Bernhardi, E.H.; van Wolferen, H.A.G.M.; Agazzi, L.; Khan, M.R.H.; Roeloffzen, C.G.H.; Wörhoff, K.; Pollnau, M.; de Ridder, R.M. Ultra-narrow-linewidth, single-frequency distributed feedback waveguide laser in $\mathrm{Al}_{2} \mathrm{O}_{3}: \mathrm{Er}^{3+}$ on silicon. Opt. Lett. 2010, 35, 2394-2396. [CrossRef]

17. Rabus, D. Integrated Ring Resonators; Springer: Heidelberg, Germany, 2007; ISBN 0342-4111.

18. Braun, R.P.; Grosskopf, G.; Meschenmoser, R.; Rohde, D.; Schmidt, F.; Villino, G. Microwave generation for bidirectional broadband mobile communications using optical sideband injection locking. Electron. Lett. 1997, 33, 1395-1396.:19970944. [CrossRef]

19. Stover, H.L.; Steier, W.H. Locking of Laser Oscillators by Light Injection. Appl. Phys. Lett. 1966, 8, 91-93. [CrossRef]

20. Bordonalli, A.C.; Walton, C.; Seeds, A.J. High-performance phase locking of wide linewidth semiconductor lasers by combined use of optical injection locking and optical phase-lock loop. J. Lightw. Technol. 1999, 17, 328-342. [CrossRef]

21. Saruwatari, M.; Nawata, K. Semiconductor laser to single-mode fiber coupler. Appl. Opt. 1979, 18, 1847-1856. [CrossRef]

22. Grebenkemper, C.J. Local Oscillator Phase Noise and its Effect on Receiver Performance. In Watkins-Johnson Company Tech-Notes; Watkins-Johnson Company: Palo Alto, CA, USA, 1981.

23. Macario, J.; Yao, P.; Shi, S.; Zablocki, A.; Harrity, C.; Martin, R.D.; Schuetz, C.A.; Prather, D.W. Full spectrum millimeter-wave modulation. Opt. Express 2012, 20, 23623-23629. [CrossRef]

24. Ito, H.; Kodama, S.; Muramoto, Y.; Furuta, T.; Nagatsuma, T.; Ishibashi, T. High-speed and high-output InP-InGaAs unitraveling-carrier photodiodes. IEEE J. Sel. Top. Quantum Electron. 2004, 10, 709-727. [CrossRef]

(C) 2019 by the authors. Licensee MDPI, Basel, Switzerland. This article is an open access article distributed under the terms and conditions of the Creative Commons Attribution (CC BY) license (http:/ / creativecommons.org/licenses/by/4.0/). 